\title{
Changing Faces of Tissue Diagnosis in the Present Era
}

\author{
$*_{\text {M F Rahman }}{ }^{1}$ \\ $1 *$ Prof. Md. Fazlur Rahman, Principal and Professor of Pathology \\ Anwer Khan Modern Medical College \\ *Corresponding Author
}

In Bangladesh, histopathology still remains the mainstay of tissue diagnosis. It provides opportunity to identify the hallmarks of malignancy such as cellular anaplasia, invasion to surrounding tissues and metastasis. It is popularly supplemented by cytologic methods where identification of cellular anaplasia is the main domain of diagnosis. Fine needle aspiration cytology (FNAC) is the most popular method where applying imaging guidance, tissue samples from deep seated lesions can also be collected and diagnosed. Other cytologic techniques are also there which not only identify the anaplastic cells, but in case of secondary tumors, they can point out even to the primary sites. But histology and cytologic methods do have certain important limitations. These sometimes fail to determine the nature of poorly differentiated tumors. Again in case of certain tumors, specially hematological malignancies, it is extremely difficult to categorize exact nature only by morphology.

\section{Immunohistochemistry}

This method now a days is gaining importance in such difficult areas. It identifies the cell products or surface markers by reacting with specific antibodies. The method has proved itself very valuable in identification of undifferentiated malignant tumors like lymphomas, melanoma, certain sarcomas and anaplastic carcinomas. The method is also of great value in case of metastatic tumors to determine its site of origin. In addition, prognosis of treatment or therapeutic prospect can be assessed by detection of certain tumor- specific molecules. ER/PR and HER2 receptors and protein products like ERBB2 are important examples.

\section{Flow Cytometry}

Flow cytometry is useful to identify cellular antigens expressed by liquid tumors especially in hematological malignancies such as B-cell and Tcell lymphomas and leukemias.

\section{Tumor Markers}

These are certain biochemical markers and are widely used as an adjunct to histological diagnosis. Here, through biochemical reactions, certain tumor-specific enzymes, hormones, oncofetal antigens, tumor-specific protein molecules etc are identified. The list of these products is increasing almost every day.

\section{Diagnostic Molecular Pathology}

The main focus of today and of the future days to diagnose malignant tumors is the wide adoption of molecular and cytogenetic diagnostic methods. Recent revolutionary progress in human genomics is reshaping the modern day approach to diagnosis and therapy. Nucleic acid based testing (DNA and RNA sequencing) is becoming crucial diagnostic tools in both inherited genetic diseases and neoplasms. Nowadays, modern technologies can rapidly sequence the entire human genome, assess epigenetic modifications genome-wide, quantify all of the RNAs expressed in a cell population, measure many proteins simultaneously and can take snapshot of all of the cells metabolites.

The main usefulness of molecular diagnostic methods are diagnosis of malignant neoplasms by differentiating monoclonal and polyclonal cell proliferation and identifying definite genetic defects, assessment of prognosis of malignant tumors by identification of poor-prognostic genetic alterations, detection of minimal residual disease after treatment thereby directing further treatment necessities, diagnosis of hereditary predisposition to cancer and guiding therapy with oncoprotein- directed drugs.

\section{Molecular Diagnostic Techniques}

- $\square$ Polymerase chain reaction (PCR):

PCR is the mainstay of molecular technologies because it forms the basis of further genomic sequencing. In this method, the DNA lying between 
designated primer sites can be exponentially amplified from as little as one original copy by using appropriate heat-stable DNA polymerase and thermal cycling. In the initial days, Sanger sequencing method was the most popular one. Later other technologies like pyrosequencing, single-base primer extension, restriction fragment length analysis (RFLA), amplicon length analysis etc were developed, each of which had their own merits and demerits. Nowadays, real-time PCR is the most popular method. Here, certain indicators like flurophore are used through which it can detect and quantify the presence of particular nucleic acid sequences during the exponential phase (real-time) of DNA amplification. PCR can also be used to amplify an RNA target sequence by reverse transcriptase (RT-PCR). Here, the RNA sequence is first converted to double stranded cDNA by using a reverse transcriptase enzyme. The remaining amplification procedure is same as DNA.

\section{- Fluorescent In-Situ Hybridization (FISH):}

FISH is based on the use of fluorescence- labeled oligonucleotide probes that specifically attach to their complementary DNA sequence target on the genome and label that region with fluorescence color (Texas red, FITCI green, acridine orange). The labeled region can then be easily visualized under a fluorescence microscope. Usually three different types of probes are used namely painting probes, centromeric probes and allele-specific probes. FISH offers great advantages over conventional cytogenetic in study of chromosomal deletions and translocations and gene amplifications (e.g; HER2/neu) as it can be performed in both dividing (metaphase) and resting (interphase) stages.

\section{- Multiplex-Ligation-Dependent Probe Amplification (MLPA):}

Here DNA hybridization, DNA ligation and PCR amplifications are blended together. It can detect deletions and duplications of any size including anomalies that are too large to be detected by PCR or too small to be identified by FISH.

\section{- Cytogenetic array technology (Microarray technology):}

Gene expression profiling using DNA microarrays holds great promise for the future of molecular diagnostics. It allows, in one assay, simultaneous assessment of the expression rate of thousands of genes in a particular sample. Thus total genomic survey can be done to detect even any subtle anomalies without any prior knowledge. The array technologies are changing day by day. In the initial times, comparative genomic hybridization (CGH) technique was used which allowed highly accurate determinations of copy number variants across the genome.

Later SNP arrays became highly popular. In developed nations, SNP arrays are routinely used to uncover copy number abnormalities especially in pediatric population. Here, the probes are designed to identify single nucleotide polymorphism (SNP) sites genome-wide to detect any type of genetic abnormalities.

\section{- Next-Generation Sequencing (NGS):}

Next Gen sequencing technologies have brought the dreams of molecular diagnostics into realities. These technologies, over the past few decades, have revolutionized biomedical research and are coming into use in the diagnosis and treatment of inherited diseases and malignancies. After advent of the complete draft of the sequence of human genome in 2003, these technologies are gradually becoming highly effective in terms of both performance and cost.

NGS are several newer DNA sequencing technologies that are capable of producing large amount of sequence data in a massively parallel manner in a short period of time. The overall steps in these methods are spatial separation, local amplification and parallel sequencing. Clinical applications of NGS DNA sequencing are wide with an aim of accurate diagnosis and targeted treatment modalities. For these objectives, targeted sequencing is the most useful method. Other technologies such as whole exome sequencing and whole genome sequencing are also used. NGS can be used to detect genetic anomalies of any size scale. As cost continues to drop, it is reasonable to expect NGS to occupy an increasingly prominent place among the diagnostic procedures. Continuing technologic advances may even extend the application further. Already third generation ('single molecule' or 'next next generation') technologies are emerging that can rapidly sequence single molecules in parallel without the need for focal amplification. 
The development of new technologies for the global molecular analysis has created a real excitement among the scientists. Even many of them have predicted that the era of histopathology is near to an end. With the advent of targeted therapies, it can be thought of that we are in the midst of a paradigm shift, where the main objective is the identification of the molecular targets rather than the histopathologic diagnosis. The diagnostic philosophy in the developed world is changing every minute. In the yet-to-be-developed world, the present day objective is to change the mindset of future medical professionals to make them updated, to take the path of research with sincere endeavors to introduce the recent technologies which can accurately diagnose tissue lesions as well as guide treatment modalities.

\section{References}

1. Collins FS, McKusick VA. Implications of the human genome project for medical science. JAMA. 2001; 285: 540-544.

2. Cazzaniga G, d'Aniello E, Corral L, Biondi A. Results of minimal residual disease (MRD) evaluation and MRD-based treatment stratification in childhood ALL. Best Pract Res Clin Haematol. 2002; 15: 623-38.

3. Cazzaniga G, Lanciotti M, Rossi V, Di Martino D, Arico M, Valsecchi MG, et al. Prospective molecular monitoring of BCR/ABL transcript in children with $\mathrm{Ph}+$ acute lymphoblastic leukaemia unravels differences in treatment response. Br J Haematol. 2002; 119: 445-453.

4. Min T, Swansbury J. Cytogenetic studies using FISH: background. Methods Mol Biol. 2003; 220: 173-191.

5. Min T. FISH techniques. Methods Mol Biol. 2003; 220: 193-212.
6. Truong K, Gibaud A, Dupont JM, Guilly MN, Soussaline F, Dutrillaux B, et al. Rapid prenatal diagnosis of Down syndrome using quantitative fluorescence in situ hybridization on interphase nuclei. Prenat Diagn. 2003; 23: $146-151$.

7. Teixeira MR. Combined classical and molecular cytogenetic analysis of cancer. Em J Cancer. 2002; 38: 1580-1584.

8. Pusztai L, Ayers M, Stec J, Hortobagyi GN. Clinical application of cDNA microarrays in oncology. Oncologist. 2003; 8: 252-258.

9. Mischel PS, Shai R, Shi T, Horvath S, Lu KV, Choe G, et al. Identification of molecular subtypes of glioblastoma by gene expression profiling. Oncogene. 2003; 22: 2361- 2373.

10. Gordon GJ, Jensen RV, Hsiao LL, Gullans SR, Blumenstock JE, Richards WG, et al. Using gene expression ratios to predict outcome among patients with mesothelioma. J natl Cancer Inst. 2003; 95: 598-605.

11. Dressman MA, Baras A, Malinowski R, Alvis LB, Kwon I, Walz TM, et al. Gene expression profiling detects gene amplification and differentiates tumor types in breast cancer. Cancer Res. 2003; 63: 2194-2199.

12. Alix-Panabieres C, Schwarzenbach H, Pantel K. Circulating tumor cells and circulating tumor DNA. Annu Rev Med. 2012; 63: 199-215.

13. Kohlmann A, Grossmann V, Haferlach T. Integration of next-generation sequencing into clinical practice: are we there yet? Semin Oncol. 2012; 39: 26-36.

14. Pao W, Lafrate AJ, Su Z. Genetically informed lung cancer medicine. J Pathol.2011; 223: 230-40. 